\title{
El juego combinativo ofensivo en el balonmano de élite: diferencias por género mediante análisis de coordenadas polares.
}

\section{The offensive combinative game in elite handball: gender differences} through polar coordinate analysis.

\section{O jogo de combinação ofensiva no handebol de elite: diferenças de género através da análise de coordenadas polares.}

\author{
Quiñones, ${ }^{1}$, Morillo-Baro, J.P. ${ }^{2}$, Reigal, R. E ${ }^{3}$, Morales-Sánchez, V. ${ }^{4}$, Vázquez-Diz, J. A. ${ }^{5}$, Hernández- \\ Mendo, A. . \\ Facultad de Psicología, Universidad de Málaga ${ }^{1,2,3,4,5,6}$
}

\begin{abstract}
RESUMEN
El objetivo de este estudio fue analizar mediante el análisis de coordenadas polares la eficacia de las conductas que se muestran durante el juego combinativo ofensivo en el balonmano de élite masculino y femenino en España. Se observaron 10 equipos pertenecientes a la Liga ASOBAL en la categoría masculina y a la Copa La Reina en la femenina. Se utilizó una herramienta observacional diseñada ad hoc mediante un sistema mixto de formatos de campo y sistema de categorías exhaustivas y mutuamente excluyentes (E/ME), formada por 12 criterios y 62 categorías con un diseño observacional Nomotético/Puntual/Multidimensional. La unidad de análisis fue el ataque posicional. Se seleccionaron cuatro conductas focales: los medios tácticos colectivos simples, la combinación de los medios tácticos, las transformaciones y el lanzamiento ventajoso. Se realizó un análisis de coordenadas polares con el software HOISAN considerando significativos los módulos $\geq 1.96$. Los resultados evidenciaron diferencias en el flujo de comportamientos entre el juego femenino y masculino. La categoría femenina con acentuadas acciones en la zona derecha del terreno. Los resultados más consistentes concuerdan con los primeros cuarenta minutos de juego y con la utilización de los medios tácticos colectivos simples y las combinaciones. En la categoría masculina se evidenció buen desempeño táctico durante todo el tiempo de juego. La combinación de los medios tácticos colectivos mostró una relación directa con el triunfo. En ambas categorías la transformación de los sistemas induce las finalizaciones en lanzamientos forzados. Las diferencias sugieren intervenir en la preparación de ambas categorías con una orientación específica hacia las mejoras en el desempeño del juego posicional combinativo.
\end{abstract}

Palabras clave: Observación sistemática, coordenadas polares, balonmano, táctica, ataque.

\section{ABSTRACT}

The aim of this study was to analyse by means of polar coordinate analysis the effectiveness of the behaviours shown during offensive combinative play in elite male and female handball in Spain. Ten teams belonging to the ASOBAL League were observed in the male category and the Copa La Reina in the female category. An observational tool 


\section{El juego combinativo ofensivo en el balonmano de élite}

designed ad hoc by means of a mixed system of field formats and a system of exhaustive and mutually exclusive categories (E/ME) was used, formed by 12 criteria and 62 categories with a Nomothetic/Punctual/Multidimensional observational design. The unit of analysis was the positional attack. Four focal behaviours were selected: simple collective tactical means, combination of tactical means, transformations and advantageous launch. An analysis of polar coordinates was carried out with the HOISAN software, considering the modules $\geq 1.96$ to be significant. The results showed differences in the flow of behaviors between the feminine and masculine game. The feminine category with accentuated actions in the right zone of the field. The most consistent results coincide with the first forty minutes of play and with the use of simple collective tactical means and combinations. In the men's category, good tactical performance was evident throughout the game. The combination of the collective tactical means showed a direct relation with the victory. In both categories the transformation of the systems induces the endings in forced throws. The differences suggest to intervene in the preparation of both categories with a specific orientation towards the improvements in the performance of the combined positional game.

Keywords: Systematic observation, polar coordinates, handball, tactics, attack.

\section{RESUMO}

O objetivo deste estudo foi analisar, através de uma análise por coordenadas polares, a eficácia dos comportamentos demonstrados durante o jogo ofensivo combinado em andebol masculino e feminino de elite em Espanha. Dez equipes pertencentes à Liga ASOBAL foram observadas na categoria masculina e a Copa La Reina na categoria feminina. Foi utilizada uma ferramenta observacional desenhada ad hoc por meio de um sistema misto de formatos de campo e um sistema de categorias exaustivas e mutuamente exclusivas (E/ME), formado por 12 critérios e 62 categorias com um desenho observacional Nomotético/Pontual/Multidimensional. A unidade de análise foi o ataque posicional. Foram selecionados quatro comportamentos focais: meios táticos coletivos simples, combinação de meios táticos, transformações e lançamento vantajoso. Uma análise das coordenadas polares foi realizada com o software HOISAN, considerando os módulos $\geq 1.96$ como significativos. Os resultados mostraram diferenças no fluxo de comportamentos entre o jogo feminino e o masculino. A categoria feminina com ações acentuadas na zona direita do campo. Os resultados mais consistentes coincidem com os primeiros quarenta minutos de jogo e com o uso de meios táticos coletivos simples e combinações. Na categoria masculina, o bom desempenho tático ficou evidente durante todo o jogo. A combinação dos meios táticos coletivos mostrou uma relação direta com a vitória. Em ambas as categorias a transformação dos sistemas induz as terminações em lançamentos forçados. As diferenças sugerem intervir na preparação de ambas as categorias com uma orientação específica para as melhorias no desempenho do jogo posicional combinado.

Palavras chave: Observação sistemática, coordenadas polares, handebol, táticas, ataque.

\section{INTRODUCCIÓN}

En la última década, se observa un crecimiento vertiginoso de investigaciones en el deporte mediante la metodología Observacional (MO). Se estudian los comportamientos deportivos en su contexto habitual; la competición, obteniendo resultados más precisos y fiables (Anguera y Hernández-Mendo, 2013, 2014; González, Botejara, Puñales, Trejo y Ruy, 2013; Morillo Baro y Hernández-Mendo, 2015; VázquezDiz, Morillo-Baro, Reigal, Morales-Sánchez y Hernández-Mendo, 2019a). Una de las potencialidades de la $\mathrm{MO}$ es el análisis de coordenadas polares; una técnica relevante para mostrar las relaciones que se establecen entre las conductas que conforman una herramienta observacional, en este caso para el ataque posicional.
Es una técnica analítica muy potente que incide en el análisis de la complejidad (Gorospe y Anguera, 2000; Morillo-Baro, Reigal y Hernández-Mendo, 2015). Vincula una determinada categoría (conducta focal o given) con todas las propias del sistema. Se apoya en un planteamiento de carácter secuencial de las sucesivas conductas ocurridas, complementándose una perspectiva prospectiva y otra retrospectiva. Esta técnica realiza una reducción drástica de los datos basada en el parámetro Zsum propuesto por Cochran (1954). Los valores que se obtienen del análisis secuencial, permiten obtener el parámetro Zsum (Zsum $=\Sigma z / \sqrt{ }$ n, donde $[\mathrm{n}]$ es el número de retardos). La distribución del parámetro Zsum tiene una $\bar{x}=0 \mathrm{y}$ una $S x=1$, permitiendo la construcción de un mapa 


\section{Quiñones, Morillo-Baro, Reigal, Morales-Sánchez, Vázquez-Diz, Hernández-Mendo.}

vectorial (Gorospe y Anguera, 2000), considerando significativos los módulos $\geq 1.96$. En un estudio realizado en el tenis, Anguera (1997) introduce la modificación del concepto de retrospectividad inicial planteada por Sackett (1980), considerando los retardos negativos como retrospectividad propia o genuina.

Deportes tan distintos como el atletismo, fútbol, taekwondo, baloncesto, esgrima o balonmano playa han utilizado el análisis de coordenadas polares por el potencial que aporta sus resultados (Aragón, Lapresa, Arana, Anguera \& Garzón, 2016; Castellano, Hernández y Haro, 2002; López-López, Menescardi, Estevan, Falcó y Hernández-Mendo, 2015; Nunes, et al., 2015; Riveiro-Bozada, et al., 2016; Tarragó, Iglesias, Lapresa y Anguera, 2016; Tarragó, et al., 2017; Vázquez-Diz, Morillo-Baro, Reigal, MoralesSánchez \& Hernández- Mendo, 2019b). El fútbol se destaca entre las disciplinas con mayores estudios realizados mediante el análisis de coordenadas polares; investigaciones dirigidas fundamentalmente al análisis de las interacciones motrices en ataque y defensa, al estudio de la dinámica de juego de jugadores élite y a las relaciones que se establecen entre los miembros de los equipos (Alday, Perea, Castellano \& Hernández-Mendo, 2009; Castañer et al., 2016; Castañer, et al., 2017; Castellano y Hernández-Mendo, 2002, 2003; Castellano, Perea \& Hernández Mendo, 2009; Díaz-Díaz, Ramos-Verde, García-Manso, Valverde-Esteve \& Arriaza-Ardiles, 2018; Echeazarra Escudero, Castellano Paulis, Usabiaga Arruabarrena y Hernández-Mendo, 2015; Maneiro \& Amatria, 2018; Maneiro, Amatria \& Anguera, 2019; Maneiro, Amatria, Moral y López, 2018).

En las investigaciones en los deportes de equipo es cada vez más sugerente el análisis de coordenadas polares para conocer las asociaciones significativas en el juego colectivo, así como las conductas de jugadores claves o determinadas posiciones de juego. El balonmano también se destaca en sus dos modalidades; en balonmano playa se estudian los comportamientos durante el ataque posicional en hombres y en mujeres (Morillo-Baro, Reigal, Hernández-Mendo, 2015; Navarro, Morillo, Reigal \& Hernández-Mendo, 2018; Vázquez-Diz, MorilloBaro, Reigal, Morales-Sánchez \& Hernández- Mendo, 2019c).
En balonmano, deporte objeto de estudio en esta investigación, la mayoría se centran en los comportamientos técnico-tácticos en la fase ofensiva; se estudia la influencia del jugador central en los resultados del juego colectivo (Flores y Anguera, 2018), las variables que influyen en la eficacia de los sistemas ofensivos (Lozano y Camerino, 2012; Lozano, Camerino e Hileno, 2016), los comportamientos de las finalizaciones de los jugadores extremos (Montoya, Moras, y Anguera, 2013), las decisiones tomadas por los árbitros en los partidos (Morillo, Reigal, Hernández-Mendo, Montaña \& Morales-Sánchez, 2017) y el juego en situaciones de ataque 2 contra 2 (Prudente, Sousa, Sequeira, López-López \& Hernández-Mendo, 2017; Sousa, Prudente, Sequeira, López-López y Hernández-Mendo, 2014).

En esta fase de juego, en el balonmano profesional, se han producido cambios relevantes en los últimos años: la velocidad en las acciones y en la toma de decisiones, y el predominio de relaciones tácticas entre dos o tres jugadores en espacios más reducidos, han derivado a una mayor utilización de los medios tácticos colectivos y a modificar la estructura de juego ofensivo con alternancia hacia un juego combinativo de medios tácticos y las transformaciones de sistemas. Morgado (2012) considera que es una forma superior de combinación, en la que participan habitualmente un número mayor de jugadores y que envuelve en su ejecución la transformación de los sistemas ofensivos (circulaciones y desdoblamientos) y las combinaciones tácticas simples (pase y va, cruce, bloqueo, pantalla, cortina y penetración sucesiva). Esta estructura de juego ha sido estudiada en equipos de balonmano de alto nivel (García, García y Aniz, 2004).

El presente estudio está dirigido a analizar el juego posicional de jugadores y jugadoras profesionales de balonmano de la liga de España del año 2018/2019; específicamente, las conductas que se muestran ante la utilización de los medios tácticos colectivos y la transformación de sistemas ofensivos. Teniendo en cuenta los supuestos anteriores, se presenta como objetivo de la investigación, analizar mediante el análisis de coordenadas polares la eficacia de las conductas que se muestran durante el juego combinativo ofensivo en el balonmano profesional masculino y femenino. 


\section{El juego combinativo ofensivo en el balonmano de élite}

\section{MATERIAL Y MÉTODOS}

\section{Diseño}

Se ha usado un diseño observacional Nomotético/Puntual/Multidimensional (N/P/M) (Anguera, Blanco-Villaseñor, Hernández-Mendo $\mathrm{y}$ Losada, 2011) situado en el cuadrante III.

\section{Participantes}

Un análisis de generalizabilidad previo (Quiñones et al., 2019) permitió estimar la totalidad de los partidos necesarios para este estudio. Se seleccionó de forma aleatoria, diez equipos profesionales de la máxima categoría de balonmano; cuatro masculinos y seis femeninos. En la categoría masculina se contó con la participación de Ángel Ximénez AVIA Puente Genil, BM. Benidorm, Bada Huesca y Frigorífico del Morrazo BM Cangas; partidos disputados durante la Liga Profesional de Balonmano Española ASOBAL 2018/2019. En la categoría femenina, Rincón Fertilidad Málaga, BM Castellón, B.M.C. Liverbank Gijón, BM Porriño, Balonmano Morvedres y Helvetia BM Alcobendas; en partidos de la Copa S.M. la Reina 2018/2019. Los partidos fueron grabados por el club local $\mathrm{y}$ posteriormente fueron solicitados $\mathrm{y}$ proporcionados para su visionado y análisis.

Se codificaron 3442 multieventos correspondientes a la categoría femenina y 3262 a la masculina, para un total de 6704 multieventos; cumpliéndose con el requisito de constancia intersesional.

\section{Instrumentos}

La herramienta de observación utilizada para este estudio ha sido diseñada ad hoc (Quiñones et al., 2019), construida con el software HOISAN, mediante un sistema mixto de formatos de campo y sistema de categorías exhaustivas y mutuamente excluyentes (E/ME) (Anguera, 1979; Castellano, 2000; Hernández-Mendo, 1996). La conforman 12 criterios (localización, marcador, minuto de juego, zona inicial de la acción, jugador que desequilibra, situación que produce el desequilibrio, técnico-táctica individual, medio táctico colectivo, ejecución de la decisión, acción defensiva, zona de finalización y resultado final) y un total de 62 categorías, que se corresponden con el orden secuencial desde que el balón es puesto en juego hasta que finaliza la acción. Todos los criterios y categorías están definidos por un núcleo categorial y su grado de plasticidad (Anguera, 1990).
Se utilizó el programa HOISAN (Hernández-Mendo, López-López, Castellano, Morales-Sánchez, y Pastrana, 2012) para codificar el flujo de conductas y posterior análisis de coordenadas polares con su representación vectorial. Se presenta la herramienta de observación con los criterios, categorías y sistema de codificación (tabla 1) y el campo de juego con las tres zonas propuestas en el estudio (figura 1).

Tabla 1. Criterios, categorías y sistema de codificación de la herramienta observacional (Quiñones et al., 2019)

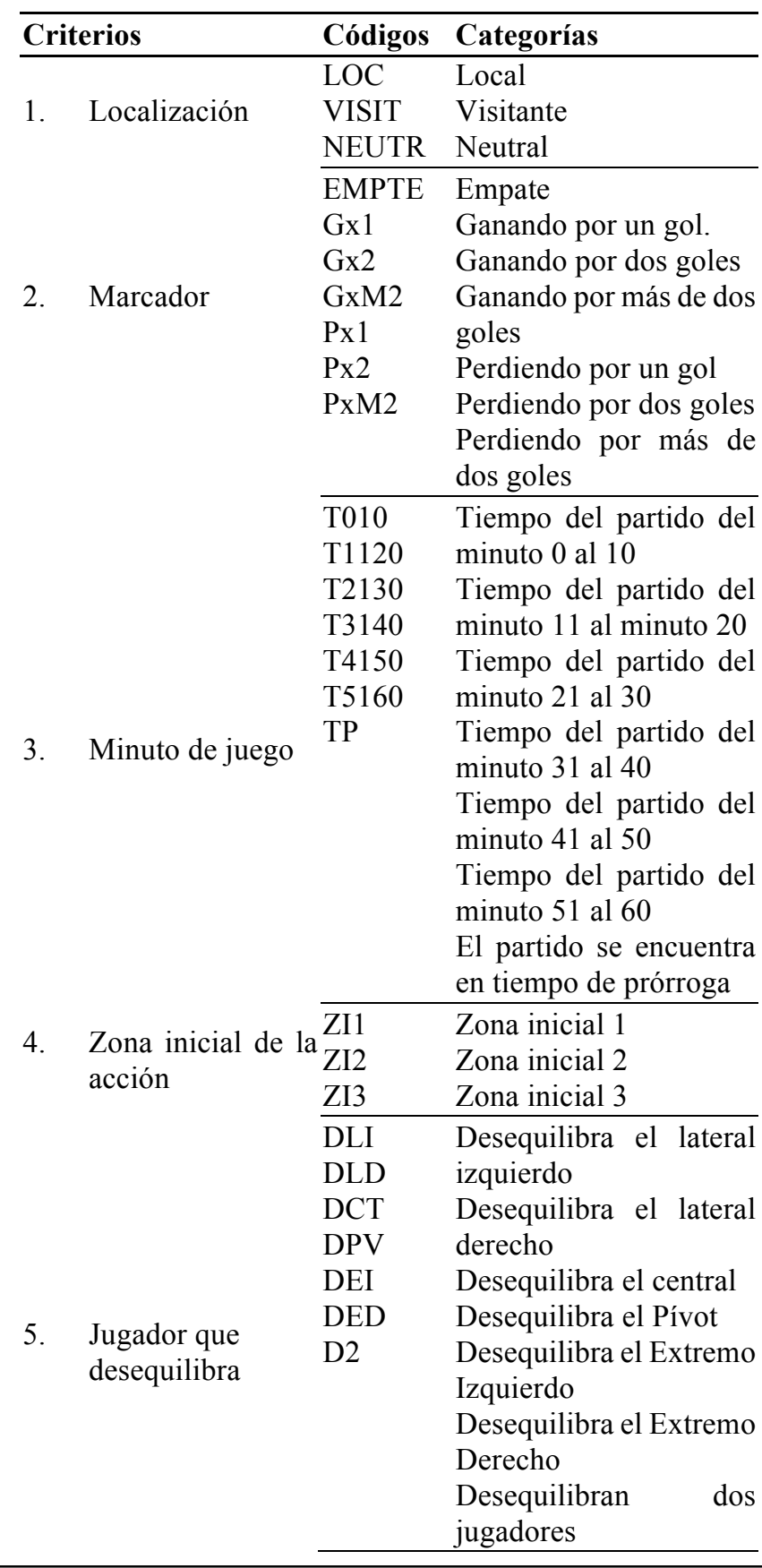




\section{Quiñones, Morillo-Baro, Reigal, Morales-Sánchez, Vázquez-Diz, Hernández-Mendo.}

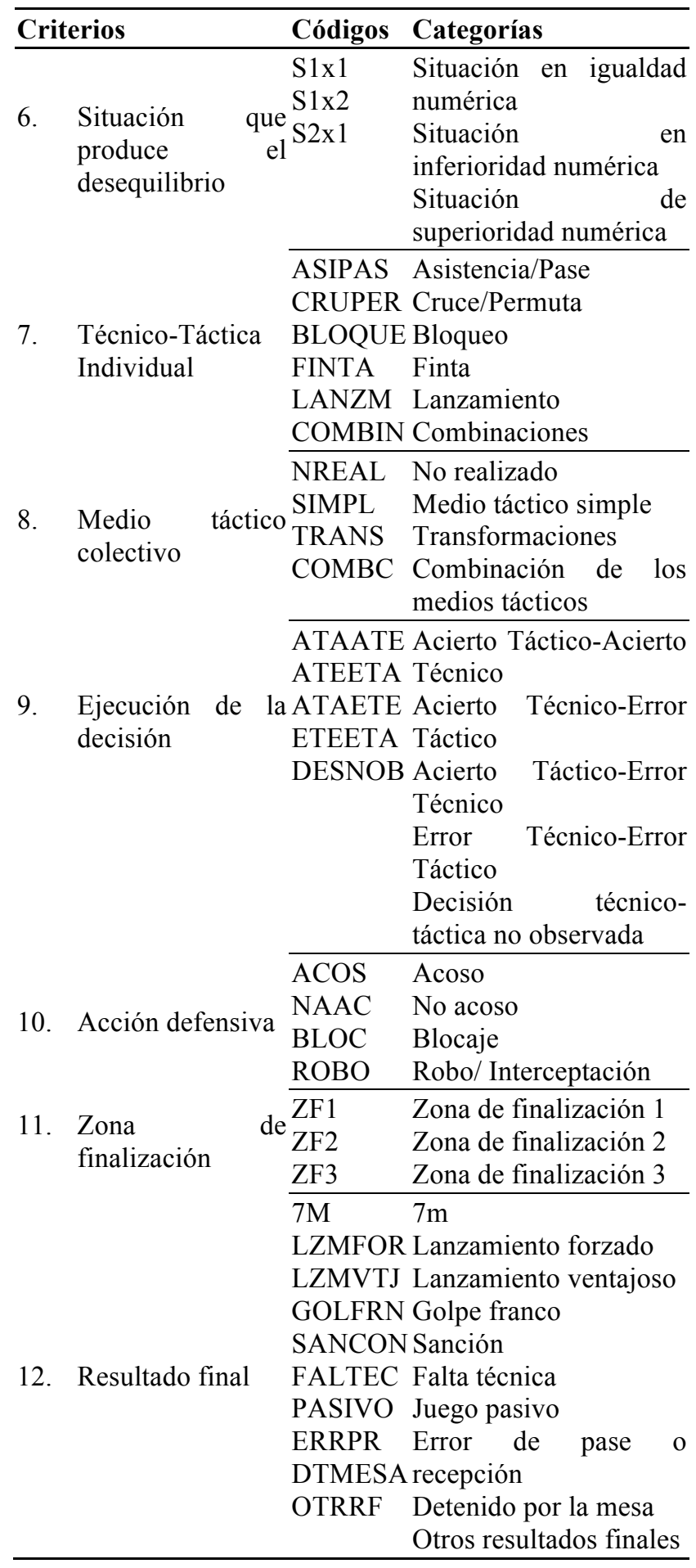

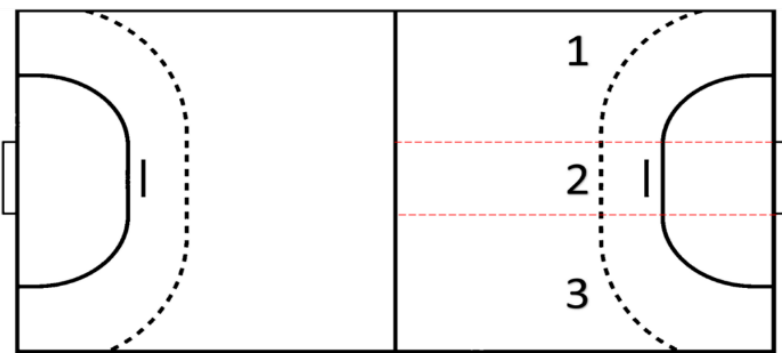

Figura 1. División de las zonas del terreno de juego (Quiñones et al., 2019)

\section{Procedimiento}

Previo al análisis de coordenadas polares, se realizó un análisis de Calidad del Dato de carácter cuantitativo con el programa HOISAN (Hernández-Mendo et al., 2012) estimando los coeficientes de correlación de Pearson, Spearman y Tau-B de Kendall; además para el concepto de asociación se estimó el índice Kappa de Cohen. La herramienta fue validada superando los análisis de Calidad del Dato (tabla 2)

Tabla 2. Resultados de los coeficientes de correlación Pearson, Spearman y Tau b de Kendall, y el índice de concordancia Kappa de Cohen de la categoría femenina y masculina (Quiñones et al., 2019)

\begin{tabular}{lllll}
\hline \multicolumn{5}{c}{ Coeficientes de Correlación } \\
\hline Coeficiente & \multicolumn{3}{l}{ Concordancia Intra } & Concordancia Inter \\
\hline \multicolumn{5}{l}{ Masculino Femenino Masculino Femenino } \\
\cline { 2 - 4 } Pearson & .99 & .97 & .98 & .98 \\
Spearman & .99 & .97 & .96 & .95 \\
$\begin{array}{l}\text { Tau b de } \\
\text { Kendall }\end{array}$ & .97 & .95 & .89 & .87 \\
$\begin{array}{l}\text { Kappa de } \\
\text { Cohen }\end{array}$ & .94 & .91 & .82 & .81 \\
\end{tabular}

Posteriormente se realizó un análisis de generalizabilidad (Cardinet, Tourneur y Allal, 1976, 1981; Cronbach, Gleser, Nanda y Rajaratnam, 1972) y se siguió el trabajo de Blanco-Villaseñor, Castellano, Hernández-Mendo, Sánchez-López y Usabiaga (2014). Con la utilización del software informático 


\section{El juego combinativo ofensivo en el balonmano de élite}

SAGT v.1.0 (Hernández-Mendo, Ramos-Pérez y Pastrana, 2012) se determinó una alta fiabilidad entre los observadores y se estimó el número mínimo de sesiones necesarios para generalizar con precisión, determinando que, con la observación de 8 partidos se obtendrían excelentes resultados. (Quiñones et al., 2019).

Finalmente, se seleccionaron cuatro categorías focales, que permiten estudiar las conductas ante la utilización de los medios tácticos colectivos y la transformación de sistemas ofensivos: medios tácticos simples (pase y va, cruce, bloqueo, pantalla, cortina y penetración sucesiva), combinación de los medios tácticos (ejecución de dos o más medios tácticos; exceptuando las circulaciones y desdoblamientos), transformaciones (ejecución de circulaciones y desdoblamientos), así como el lanzamiento ventajoso (lanzamiento a puerta con clara posibilidad de ejecución). El resto de las categorías incluidas en la herramienta observacional fueron conductas condicionales, excepto las pertenecientes al mismo criterio. Empleando el software HOISAN, se realizó el análisis de coordenadas polares; determinando las relaciones excitatorias e inhibitorias entre las conductas focales y las conductas condicionales seleccionadas. En este análisis fueron consideradas significativa $(\mathrm{p}<0.05)$; o sea, las relaciones con un radio o longitud del vector de $\geq 1.96$.

Caracterización de cada cuadrante (Castellano y Hernández-Mendo, 2003):

Cuadrante $\mathrm{I}[+,+]$ : La conducta criterio se excita con respecto a la conducta de apareo en perspectiva retrospectiva y prospectiva.
Cuadrante II $[-,+]$ : La conducta criterio tiene una relación con respecto a la de apareo de excitación en perspectiva retrospectiva y de inhibición en perspectiva prospectiva.

Cuadrante III [-,-]: La conducta criterio tiene una relación con respecto a la de apareo de inhibición en perspectiva retrospectiva y prospectiva.

Cuadrante IV [+,-]: La conducta criterio tiene una relación con la conducta de apareo de excitación en perspectiva prospectiva y de inhibición en perspectiva retrospectiva.

Las conductas seleccionadas como focales para este estudio han sido los medios tácticos colectivos: simples, combinación de los medios tácticos y transformación, además de la conducta lanzamiento ventajoso. Se tomaron como conductas de apareo, en cada caso, todas las categorías de la herramienta que no forman parte del propio criterio seleccionado como conducta focal.

\section{RESULTADOS}

A continuación se presentan los resultados del análisis de coordenadas polares para las cuatro conductas focales seleccionadas (SIMPL, COMBC, TRANS y LZMVTJ) y las asociaciones significativas con el resto de las conductas de la herramienta de observación en la categoría femenina y masculina.

La tabla 3 muestra las asociaciones significativas de las conductas focales medios tácticos simples (SIMPL) y la combinación de medios tácticos (COMBC) con las conductas de apareo en la categoría femenina y masculina.

Tabla 3. Relaciones significativas en cada uno de los cuadrantes entre las conductas focales SIMPL y COMBC

\begin{tabular}{llllllll}
\hline \multirow{2}{*}{$\begin{array}{c}\text { Conducta } \\
\text { focal }\end{array}$} & C & \multicolumn{3}{c}{ Femenino } & \multicolumn{2}{c}{ Masculino } \\
\cline { 3 - 7 } & & $\begin{array}{l}\text { Conducta } \\
\text { de apareo }\end{array}$ & $\begin{array}{l}\text { Módulo } \\
\text { Vector }\end{array}$ & A.T & $\begin{array}{l}\text { Conducta } \\
\text { de apareo }\end{array}$ & $\begin{array}{l}\text { Módulo } \\
\text { Vector }\end{array}$ & A.T. \\
\hline & & & & & \\
& & GxM2 & 2.41 & 53.09 & LOC & 4.77 & 62.11 \\
& & T1120 & 3.25 & 32.06 & EMPTE & 2.10 & 7.30 \\
& I & T3140 & 2.16 & 82.14 & GxM2 & 5.82 & 34.61 \\
SIMPL & & & & GOLFRN & 2.37 & 63.88 \\
\hline
\end{tabular}




\section{Quiñones, Morillo-Baro, Reigal, Morales-Sánchez, Vázquez-Diz, Hernández-Mendo.}

\begin{tabular}{|c|c|c|c|c|c|c|c|}
\hline & & $\mathrm{Gx} 2$ & 368 & 209.14 & VISIT & 4.92 & 238.96 \\
\hline & III & $\mathrm{T} 4150$ & 2.29 & 261.30 & Gx1 & 3.28 & 198.74 \\
\hline & & T5160 & 2.72 & 225.59 & $\mathrm{Gx} 2$ & 4.47 & 215.71 \\
\hline & IV & EMPTE & 2.14 & 297.58 & LZMVTJ & 3.62 & 275.20 \\
\hline \multirow{13}{*}{ COMBC } & \multirow{3}{*}{ I } & LOC & 5.14 & 42.79 & Gx1 & 3.40 & 9.59 \\
\hline & & GxM2 & 2.46 & 20.24 & LZMVTJ & 4.14 & 84.84 \\
\hline & & $\mathrm{T} 2130$ & 2.57 & 64.26 & & & \\
\hline & \multirow{2}{*}{ II } & EMPTE & 1.98 & 121.37 & ZF3 & 1.98 & 138.57 \\
\hline & & & & & FALTEC & 2.08 & 157.30 \\
\hline & \multirow{5}{*}{ III } & VISIT & 5.08 & 223.37 & EMPTE & 3.03 & 194.06 \\
\hline & & Px1 & 4.06 & 220.07 & & & \\
\hline & & Px2 & 5.44 & 201.57 & & & \\
\hline & & T3140 & 4.63 & 244.03 & & & \\
\hline & & GOLFRN & 3.24 & 235.46 & & & \\
\hline & \multirow{3}{*}{ IV } & FALTEC & 2.46 & 319.02 & $\mathrm{Gx} 2$ & 2.23 & 296.34 \\
\hline & & $\mathrm{Gx} 2$ & 3.78 & 355.24 & ZF2 & 2.41 & 317.61 \\
\hline & & & & & GOLFRN & 2.34 & 277.09 \\
\hline
\end{tabular}

En el cuadrante I se visualizan las relaciones mutuamente excitatorias en perspectiva prospectiva y retrospectiva entre la conducta focal y las conductas de apareo. La conducta focal medios tácticos simples (SIMPL) en la categoría femenina se relaciona con dos conductas de apareo del criterio minuto de juego: minuto del 11 al 20 (T1120) y del 31 al 40 (T3140). En la categoría masculina se relaciona con el marcador en empate (EMPTE), con golpe franco (GOLFRN) y con la categoría local (LOC). En ambos sexos coincide la relación de la conducta ganando por más de dos goles (GxM2).

En este mismo cuadrante, pero de la conducta focal combinación de medios tácticos (COMBC) en la categoría femenina se presenta una relación con las conductas de apareo local (LOC), ganando por más de dos goles (GxM2) y el minuto de juego del 21 al 30 (T2130); y en la masculina con dos conductas de apareo: ganando por un gol $(\mathrm{Gx} 1)$ y con lanzamiento ventajoso (LZMVTJ).

En el cuadrante II las asociaciones significativas de la conducta focal respecto a las conductas de apareo son de inhibición en perspectiva prospectiva y de excitación en perspectiva retrospectiva. Los resultados obtenidos en la conducta medios tácticos simple (SIMPL) en la categoría femenina excitan al marcador perdiendo por más de dos goles (PxM2) y en la masculina a la zona de finalización dos (ZF2). A diferencia de la conducta focal combinación de los medios tácticos (COMBC) que en la categoría femenina excita el empate (EMPTE) y en la masculina a la zona de finalización tres $(\mathrm{ZF} 3)$ y a la falta técnica (FALTEC).

El cuadrante III representa las relaciones mutuamente inhibitorias en perspectiva prospectiva y retrospectiva entre la conducta focal y las conductas de apareo. Cuando la conducta focal es medios tácticos simples (SIMPL) aparece la relación con la categoría ganando por dos goles (Gx2) en ambos sexos. A diferencia, en la conducta focal combinación de los medios tácticos (COMBC), no existen coincidencias en las relaciones; la mutua inhibición se presenta de manera significativa y con gran intensidad con cinco categorías en el sexo femenino y solo con el empate (EMPTE) en el sexo masculino.

En el cuadrante IV la conducta focal tiene una relación de excitación en prospectiva y de inhibición en retrospectiva con las conductas de apareo. La conducta focal medios tácticos simples (SIMPL) en la categoría femenina inhiben el empate (EMPTE), y en la categoría masculina al lanzamiento ventajoso (LZMVTJ). Mientras que, cuando se excita la conducta focal combinación de los medios tácticos (COMBC) inhibe la falta técnica en los equipos femeninos y el golpe franco en los equipos masculinos; coincide esta relación cuando ganan ambos equipos por dos goles. Los resultados de la conducta focal SIMPL se representan en la figura 2 y la conducta $\mathrm{COMBC}$ en la figura 3. 


\section{El juego combinativo ofensivo en el balonmano de élite}

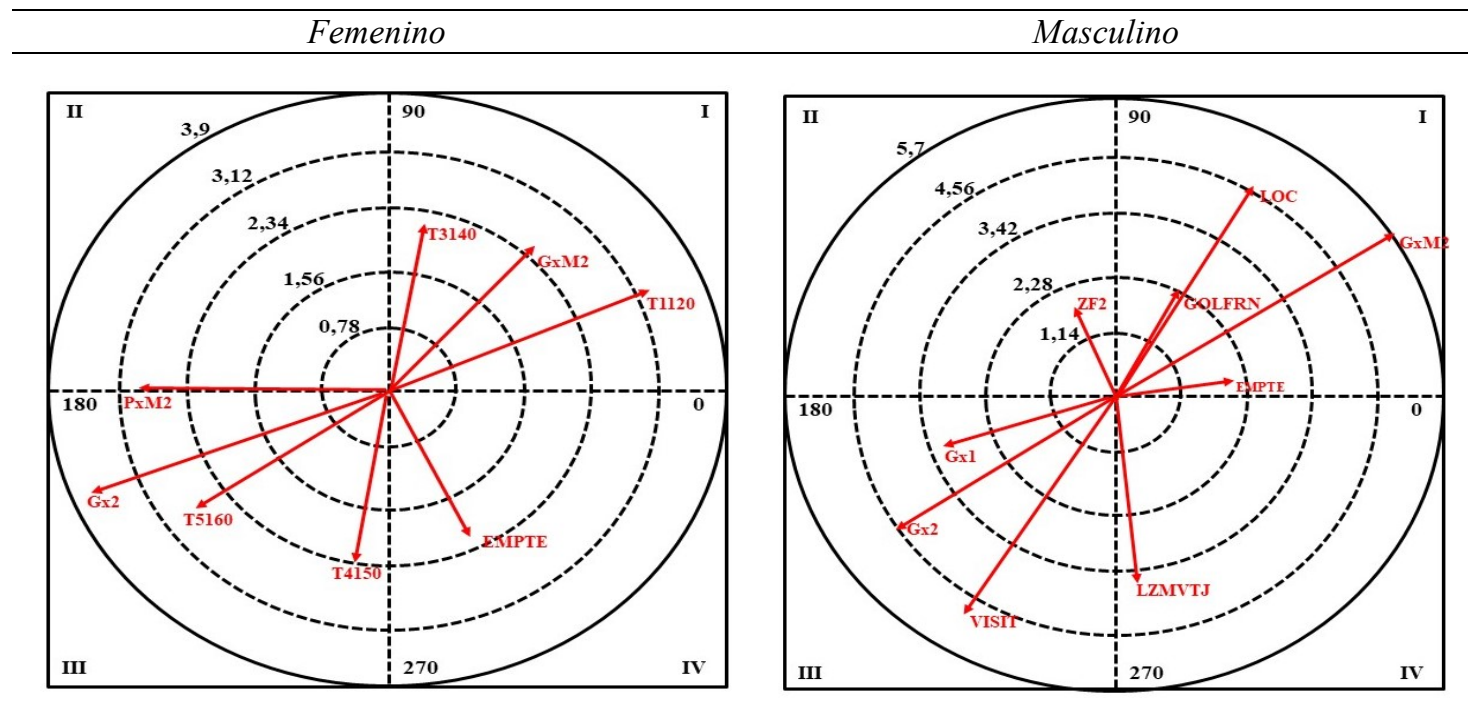

Figura 2. Representación gráfica del análisis de coordenadas polares en ambas categorías para la conducta focal SIMPL

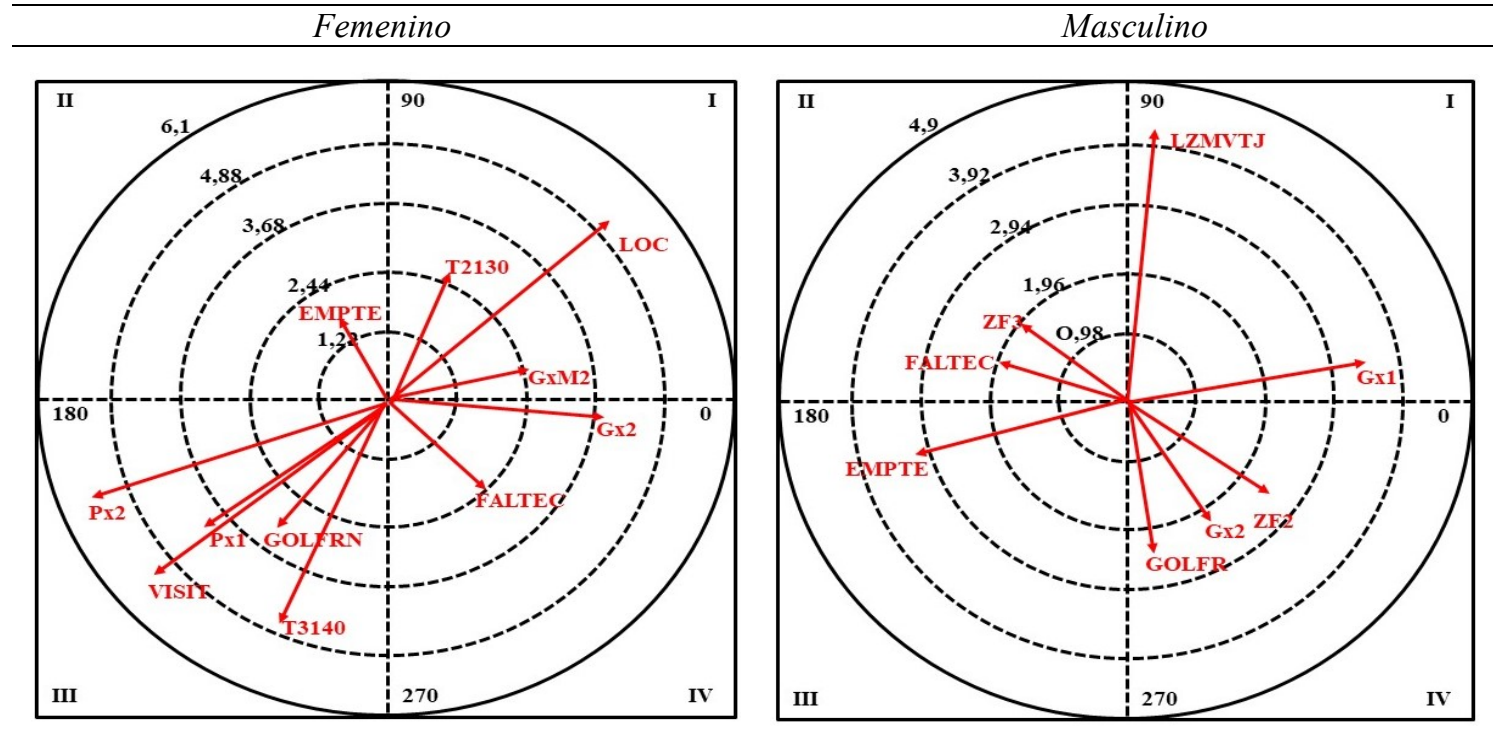

Figura 3. Representación gráfica del análisis de coordenadas polares en ambas categorías para la conducta focal COMBC

En la tabla 4 se muestran los resultados de las coordenadas polares de la conducta focal transformaciones (TRANS) para la categoría femenina y masculina. 


\section{Quiñones, Morillo-Baro, Reigal, Morales-Sánchez, Vázquez-Diz, Hernández-Mendo.}

Tabla 4. Relaciones significativas de cada cuadrante entre la conducta focal TRANS y las conductas de apareo en las dos categoría

\begin{tabular}{|c|c|c|c|c|c|c|c|}
\hline \multirow[b]{2}{*}{$\begin{array}{l}\text { Conducta } \\
\text { focal }\end{array}$} & \multirow[b]{2}{*}{$\mathrm{C}$} & \multicolumn{3}{|l|}{ Femenino } & \multicolumn{3}{|l|}{ Masculino } \\
\hline & & $\begin{array}{l}\text { Conducta de } \\
\text { apareo }\end{array}$ & $\begin{array}{l}\text { Módulo } \\
\text { Vector }\end{array}$ & A.T. & $\begin{array}{l}\text { Conducta de } \\
\text { apareo }\end{array}$ & $\begin{array}{l}\text { Módulo } \\
\text { Vector }\end{array}$ & A.T. \\
\hline \multirow{13}{*}{ TRANS } & \multirow{5}{*}{ I } & VISIT & 7.10 & 40.11 & VISIT & 8.12 & 4.46 \\
\hline & & Px1 & 2.19 & 64.01 & Gx2 & 5.14 & 38.47 \\
\hline & & Px2 & 3.49 & 26.49 & LZMFOR & 2.48 & 45.05 \\
\hline & & PxM2 & 4.84 & 36.08 & & & \\
\hline & & LZMFOR & 2.41 & 55.39 & & & \\
\hline & \multirow{2}{*}{ II } & GX2 & 4.91 & 91.14 & EMPTE & 2.43 & 96.97 \\
\hline & & $\mathrm{T} 4150$ & 2.26 & 124.85 & PxM2 & 1.97 & 136.57 \\
\hline & \multirow{2}{*}{ III } & LOC & 6.97 & 220.97 & LOC & 8.10 & 222.60 \\
\hline & & GxM2 & 9.20 & 227.64 & GxM2 & 6.39 & 225.00 \\
\hline & \multirow{4}{*}{ IV } & DED & 2.67 & 338.42 & $7 \mathrm{M}$ & 2.05 & 295.66 \\
\hline & & Gx1 & 2.22 & 336.05 & FALTEC & 2.70 & 343.57 \\
\hline & & T5160 & 2.71 & 338.32 & & & \\
\hline & & ZF2 & 1.96 & 335.55 & & & \\
\hline
\end{tabular}

En el cuadrante I, las relaciones mutuamente excitatorias en perspectiva prospectiva y retrospectiva de la conducta focal transformaciones coinciden en ambas categorías con el lanzamiento forzado (LZMFOR) y con la condición de visitante (VISIT); esta última muestra una alta intensidad en ambos sexos. Las restantes relaciones asociativas significativas aparecen con cuatro categorías del criterio marcador del juego: perdiendo por 1 gol (Px1), perdiendo por 2 goles ( $\mathrm{Px} 2)$, perdiendo por más de dos goles (PxM2) y ganando por dos goles (Gx2).

En el cuadrante II las relaciones de inhibición en perspectiva prospectiva y de excitación en perspectiva retrospectiva de la conducta focal transformaciones, en la categoría femenina se muestran con las conductas ganando por dos goles y tiempo de juego del minuto 41 al 50, y en la categoría masculina con las conductas empate y perdiendo por más de dos goles.
La mayor intensidad en las relaciones ocurre en la categoría ganando por dos goles de los partidos femeninos.

Las relaciones de mutua inhibición que se presentan en el cuadrante III muestran resultados coincidentes en ambos sexos con las categorías local y ganando por más de dos goles; esta relación se presenta con una alta intensidad.

El cuarto cuadrante de la conducta focal TRANS, en la categoría femenina inhibe la ocurrencia de cuatro conductas: desequilibra el extremo derecho (DED), ganando por un gol (Gx1), Tiempo del partido del minuto 51 al 60 (T5160) y zona de finalización dos (ZF2). Por su parte, en la categoría masculina, inhibe dos resultados de finalización: 7M y FALTEC. Los resultados se representan en la figura 4 . 


\section{El juego combinativo ofensivo en el balonmano de élite}

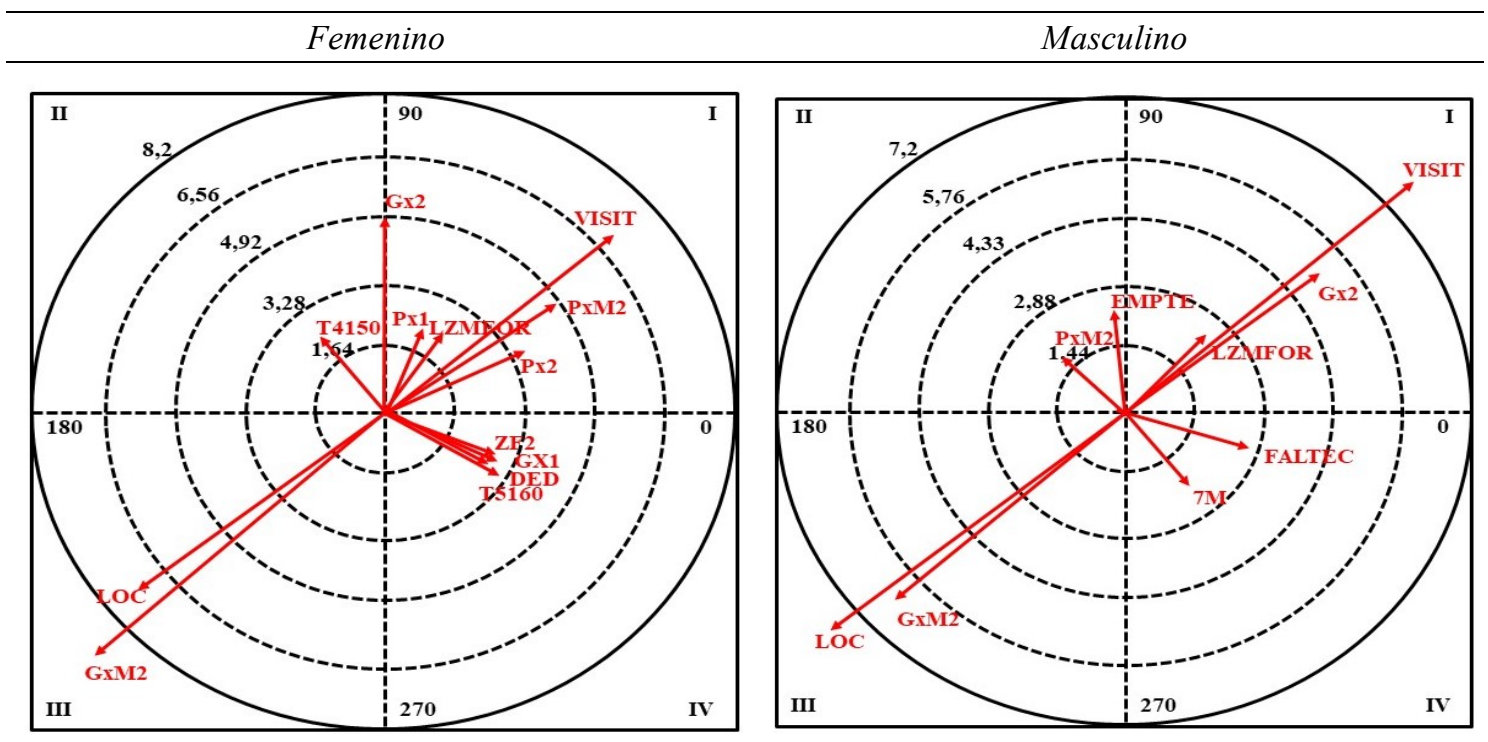

Figura 4. Representación gráfica del análisis de coordenadas polares en ambas categorías para la conducta focal TRANS

En la tabla 5 se presentan las relaciones entre la conducta focal lanzamiento ventajoso (LZMVTJ) y las conductas de apareo en la categoría femenina y masculina.

Tabla 5. Relaciones significativas de cada cuadrantes entre la conducta focal LZMVTJ y las conductas de apareo en las dos categorías

\begin{tabular}{|c|c|c|c|c|c|c|c|}
\hline \multirow[b]{2}{*}{$\begin{array}{l}\text { Conducta } \\
\text { focal }\end{array}$} & \multirow[b]{2}{*}{$\mathrm{C}$} & \multicolumn{3}{|l|}{ Femenino } & \multicolumn{3}{|l|}{ Masculino } \\
\hline & & $\begin{array}{l}\text { Conducta de } \\
\text { apareo }\end{array}$ & $\begin{array}{l}\text { Módulo } \\
\text { Vector }\end{array}$ & A.T. & $\begin{array}{l}\text { Conducta de } \\
\text { apareo }\end{array}$ & $\begin{array}{l}\text { Módulo } \\
\text { Vector }\end{array}$ & A.T. \\
\hline \multirow{15}{*}{ LZMVTJ } & \multirow{6}{*}{ I } & GxM2 & 3.25 & 18.99 & COMBC & 4.14 & 5.16 \\
\hline & & PxM2 & 4.68 & 67.73 & Gx1 & 3.33 & 257.18 \\
\hline & & T010 & 2.75 & 41.95 & Px2 & 2.00 & 82.45 \\
\hline & & T1120 & 3.94 & 36.60 & & & \\
\hline & & $\mathrm{T} 2130$ & 2.63 & 56.03 & & & \\
\hline & & DLD & 2.14 & 6.830 & & & \\
\hline & \multirow{3}{*}{ II } & DCT & 2.31 & 152.90 & SIMPL & 3.62 & 174.80 \\
\hline & & & & & ZI3 & 1.99 & 107.61 \\
\hline & & & & & ZF3 & 2.65 & 168.64 \\
\hline & \multirow{5}{*}{ III } & EMPTE & 3.82 & 191.75 & EMPTE & 3.33 & 257.18 \\
\hline & & $\mathrm{Px} 2$ & 4.17 & 213.93 & GxM2 & 2.48 & 224.63 \\
\hline & & T3140 & 3.43 & 228.82 & PxM2 & 2.42 & 187.29 \\
\hline & & T4150 & 2.38 & 220.78 & & & \\
\hline & & T5160 & 2.81 & 220.13 & & & \\
\hline & IV & Gx2 & 2.51 & 320.60 & & & \\
\hline
\end{tabular}

En el primer cuadrante, las relaciones de mutua excitación de la conducta focal Lanzamiento ventajoso ocurren con nueve categorías; en su mayoría pertenecientes a los criterios marcador y minuto de juego. En los partidos femeninos la relaciones se muestran con GxM2, PxM2, T010, T1120, T2130 y DLD. La mayor intensidad ocurre con la conducta perdiendo por más de dos goles con un módulo de 


\section{Quiñones, Morillo-Baro, Reigal, Morales-Sánchez, Vázquez-Diz, Hernández-Mendo.}

4.68. En los partidos masculinos las relaciones de mutua excitación se dan con las categorías COMBC, Gx1 y Px2. La mayor intensidad se presenta en la combinación de los medios con un módulo de 4.14.

En el segundo cuadrante, se excita la presencia del desequilibrio del jugador central en la categoría femenina, y en la masculina excita tres conductas: los medios tácticos simple (SIMPL) y las zonas inicial y final tres (ZI3 y ZF3).
En el tercer cuadrante, las conductas de apareo que ocurren en la categoría femenina y la masculina pertenecen a los criterios marcador y minuto de juego; coincidiendo el empate (EMPTE) en ambas categorías. Resulta significativa la intensidad de la relación con Px2. Por último, en el cuarto cuadrante no se producen relaciones en la categoría masculina; la relación se establece en la categoría femenina con la conducta de apareo ganando por dos goles (Gx2). Los resultados se presentan en los gráficos de la figura 5.

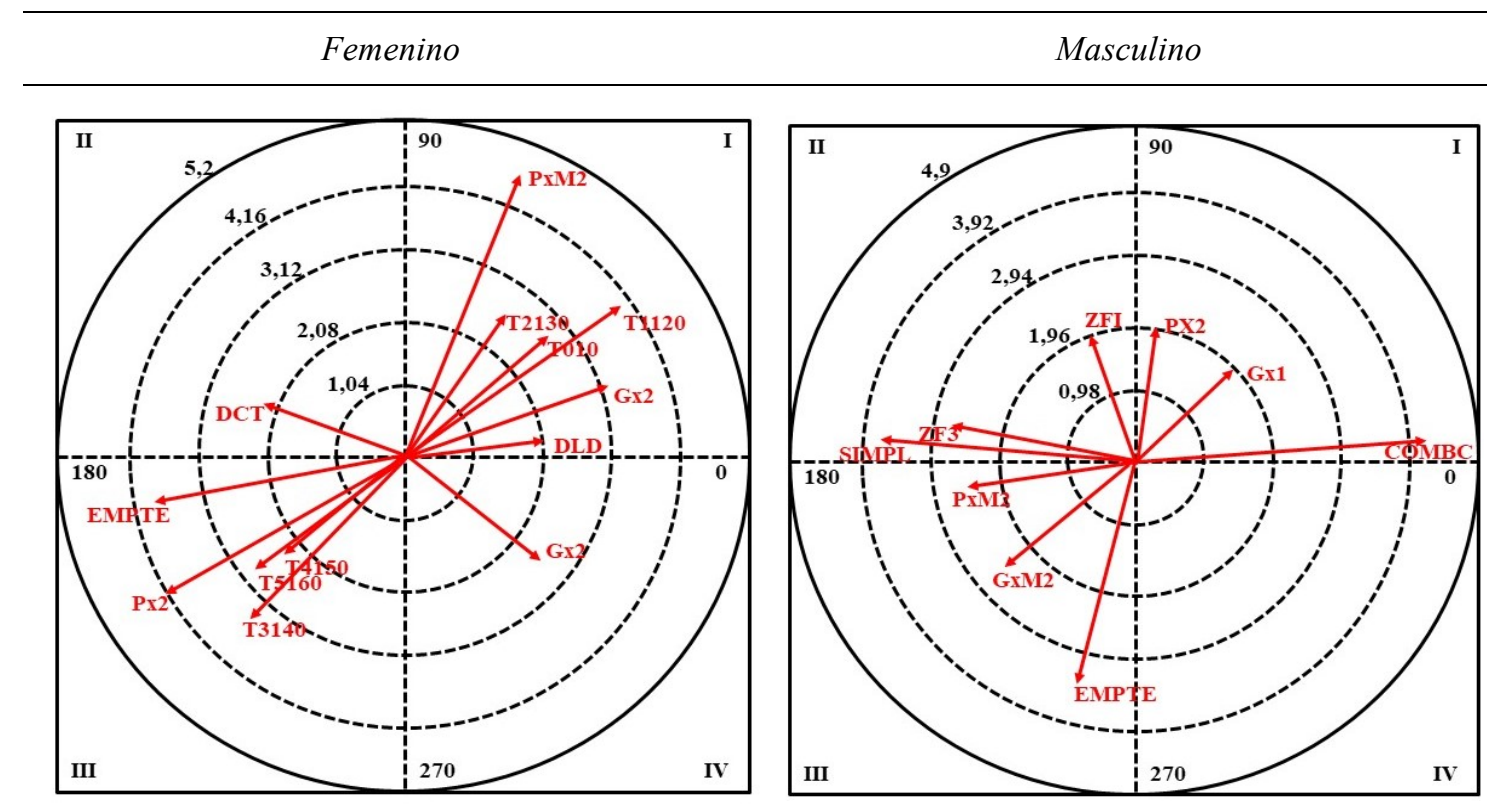

Figura 5. Representación gráfica del análisis de coordenadas polares en ambas categorías para la conducta focal LZMVTJ

\section{DISCUSIÓN}

El objetivo de la investigación fue analizar mediante el análisis de coordenadas polares la eficacia de las conductas que se muestran en el juego combinativo ofensivo en el balonmano profesional masculino y femenino. Los hallazgos en este estudio han permitido identificar diferencias en el flujo de comportamientos entre la categoría femenina y masculina. El juego femenino muestra mayor utilización de los medios tácticos colectivos simples y sus combinaciones en la primera mitad de los partidos y asociados a las ventajas en el marcador, contrario a la transformación de los sistemas con una marcada utilización en los últimos minutos de juego. En cambio, en la categoría masculina se relacionan ambos recursos tácticos con los dos períodos del partido, sin embargo, las conductas del juego más eficaces se alcanzaron con la utilización de los medios tácticos simples y la combinación de los medios tácticos. Tanto en el juego femenino como en el masculino se manifiesta una relación entre la transformación de los sistemas y la ejecución de los lanzamientos en condiciones forzadas.

En primer lugar, en los partidos femeninos la utilización de los medios tácticos colectivos (pase y va, cruce, bloqueo, pantalla, cortina y penetración sucesiva) se asocian al inicio de cada período de juego, cuando prevalecen niveles óptimos de rendimiento físico. Se relacionan a la ventaja, la desventaja y a los períodos de empate en el marcador, por lo que se 


\section{El juego combinativo ofensivo en el balonmano de élite}

interpreta que puede estar relacionado con el éxito. De acuerdo a los resultados obtenidos, Prudente et al. (2017) corrobora las relaciones entre las acciones tácticas y la evolución de la puntuación en todos los parciales. La combinación de los medios tácticos colectivos es un recurso que a este nivel se aprovecha para desarrollar un juego creativo y obtener las mayores ventajas. Las conductas que muestran resultados más eficaces se manifiestan fundamentalmente en la primera mitad del partido, varias de estas acciones culminan en golpes francos y faltas técnicas; estas últimas se traducen en pérdidas del balón; coincidentes con Vázquez-Diz et al. (2019c) donde se evidencian asociaciones de errores y faltas técnicas en los últimos minutos del partido. Contrariamente, Prudente et al. (2017) encuentran estos resultados principalmente al final de la primera mitad.

Al analizar el juego en transformación (utilización de circulaciones y desdoblamientos) se manifiesta una relación con la desventaja en el marcador y la condición de visitante. De igual modo hay una correspondencia de estas acciones tácticas a la iniciación por las jugadoras extremos derechos, con finalización en la zona central. La vinculación significativa con la conducta que describe un lanzamiento ejecutado en condiciones forzadas, sin clara posibilidad de gol, es contrario a lo que se pretende al realizar un ataque elaborado. En consecuencia, se señala que el juego en transformación debe tener colaboración de todas las jugadoras y ser imprevisto para la defensa. A este nivel deben entrenarse las iniciativas de las jugadoras especialistas en interpretar cuando las condiciones defensivas están creadas para realizar una transformación; incluso, sin indicación de la jugadora central. En relación, García, Aniz, Arellano, Domínguez y García (2004) coinciden en que al realizar las transformaciones se necesita la colaboración, tanto de la primera como de la segunda línea ofensiva, por lo tanto, al transformar el sistema a un juego con cuatro segundas líneas ofensivas debe haber participación tanto en tareas de finalización como en acciones de creación; de esta manera se garantiza el desequilibrio defensivo inicial y la continuidad del juego. Los resultados encontrados en las relaciones han identificado un área de mejora en esta categoría.
En la categoría masculina se muestra que los medios tácticos colectivos simples y sus combinaciones tienen grandes relaciones significativas con los períodos de empates y ventaja en el marcador; coincidentes con Lozano, Camerino e Hileno, (2016b) y Prudente et al. (2017) donde el estado del marcador condiciona la utilización de los medios tácticos, además de considerar que la realización de uno u otro depende de las características del contrario y del sistema defensivo de los rivales. De acuerdo a otros resultados se evidencia varias finalizaciones; el golpe franco y las faltas técnicas, coincidentes con otros estudios (González, 2012; Meletakos, Vagenas \& Bayios, 2011; Teles, 2011) y con la estructura ofensiva hallada por Lozano, Camerino e Hileno (2016a).

Otro resultado de finalización es el lanzamiento ventajoso, es decir, lanzamiento con clara posibilidad de gol, en su mayoría ejecutados por la zona central del campo. Las asociaciones encontradas en este estudio entre las finalizaciones y las condiciones del marcador contradicen parcialmente los resultados de Prudente et al. (2017) al mostrar la utilización de acciones menos arriesgadas para evitar la pérdida del balón, principalmente por faltas técnicas.

La realización de transformaciones en los partidos masculinos se relaciona con todas las posibles situaciones del marcador: ventaja, desventaja y empate. Se constatan como finalizaciones, fundamentalmente, los lanzamientos forzados, las faltas técnicas y los lanzamientos de 7 metros; datos esperados, considerando que al realizar estas acciones tácticas obligan a la defensa a perder profundidad y las acciones ofensivas se desarrollan en contacto directo con la defensa, entre las líneas de 6 y 9 metros. En otros estudios, además, se ha obtenido como finalización el golpe franco (García, García e Inarejos, 2002; García, et al., 2004). Estas asociaciones identificadas permiten estimar las ventajas que se obtienen al realizar la transformación de sistemas. Las acciones esperadas por la defensa, permiten aprovechar los obligados cambios en los sistemas defensivos para encontrar distintas opciones de finalización, incluso, de manera sorpresiva desde la segunda línea ofensiva.

Fueron analizadas además las relaciones con el lanzamiento ventajoso. En la categoría femenina se manifiestan en la primera mitad de los partidos. Situaciones provocadas por el desequilibrio de las 


\section{Quiñones, Morillo-Baro, Reigal, Morales-Sánchez, Vázquez-Diz, Hernández-Mendo.}

jugadoras centrales y laterales derechos, poniendo de manifiesto un juego focalizado en una parte del terreno, por lo que la defensa se prepara para impedir o limitar las acciones. También con el marcador de juego, tanto en ventaja como en desventaja, lo cual refleja que el marcador no condicionaría la calidad de la ejecución del lanzamiento. A diferencia de estos resultados, en la categoría masculina la mayoría de los lanzamientos en condiciones ventajosas ocurren ante la utilización de la combinación de los medios tácticos colectivos, mostrando el éxito de las acciones grupales. Se manifiesta como un recurso utilizado con gran seguridad en los partidos. Coincidentes con la categoría femenina se obtuvieron relaciones significativas con el marcador, además de corroborar que las mejores condiciones de lanzamientos se producen con iniciaciones y finalizaciones en la zona derecha del campo de juego.

Este estudio ha permitido analizar la eficacia del juego combinativo ofensivo en las dos categorías. Los resultados encontrados han aportado informaciones difíciles de obtener sin el análisis de coordenadas polares. Particularmente, en la categoría femenina se limita el análisis con otras investigaciones de este género, puesto que, la mayoría de los estudios del juego posicional en balonmano han sido dirigidos a equipos masculinos. No se obtuvieron relaciones significativas con algunas conductas; por ejemplo, las pertenecientes a las acciones defensivas y a la ejecución de la decisión; sin embargo los datos obtenidos han permitido analizar las diferencias y similitudes del juego posicional femenino $y$ masculino.

\section{APLICACIONES PRÁCTICAS}

De la investigación emergen como posibles aplicaciones prácticas: acceder a las informaciones aportadas del análisis de coordenadas polares, permitiendo a entrenadores y preparadores de equipos de élite conocer las posibles conductas que ocurren durante el juego combinativo ofensivo, así como obtener rastros de conductas de los sistemas de juego de los equipos adversarios, con el objetivo de mejorar la eficacia de estas acciones en competición. Diseñar en las sesiones de entrenamiento estructuras de ataque, principalmente del juego con transformación de sistemas, con responsabilidades repartidas tanto para la primera como segunda línea ofensiva, que faciliten la continuidad del juego y las ventajas en las finalizaciones. De igual modo, entrenar desde las categorías de iniciación estructuras de ataque en transformación sin la indicación del central, sino con iniciativas de los participantes en situaciones favorables para desarrollar esta estructura de ataque.

\section{REFERENCIAS}

1. Alday, L., Perea, A., Castellano, J. \& Hernández-Mendo, A. (2009). Polar Coordinate Analysis of the soccer World Championships using Matlab. In A. Hökelmann, K. Witte \& P. O’Donoghe (Eds.) Current trends in performance analysis ( $\mathrm{pp}$. 337-344). Shaker Verlag: Aachen (Germany).

2. Anguera, M. T. (1979). Observación de la conducta espacial. Comunicación presentada al VI Congreso Nacional de Psicología, Pamplona, España.

3. Anguera, M. T. (1990). Metodología observacional. In J. Arnau, M. T. Anguera, \& J. Gómez Benito (Eds.), Metodología de la investigación en ciencias del comportamiento (pp. 125-236). Murcia: Universidad de Murcia.

4. Anguera, M.T. (1997). From prospective patterns in behavior to joint analysis with a retrospective perspective. Colloque sur invitation «Méthodologie d'analyse des interactions sociales». Paris: Université de la Sorbonne.

5. Anguera, M. T., Blanco, A., HernándezMendo, A. y Losada, J. L. (2011). Diseños observacionales: ajuste y aplicación en psicología del deporte. Cuadernos de Psicología del Deporte, 11 (2), 63-76. http://dx.doi.org/10.4321/S157884232015000100002

6. Anguera, M. T., y Hernández-Mendo, A. (2013). La metodología observacional en el ámbito del deporte. E-balonmano.com: Revista de Ciencias del Deporte 9(3), 135- 


\section{El juego combinativo ofensivo en el balonmano de élite}

160. Recuperado de www.ebalonmano.com/ojs/index.php/revista/about

7. Anguera, M. T. y Hernández-Mendo, A. (2014). Metodología observacional y psicología del deporte: Estado de la cuestión. Revista de Psicología del Deporte, 23(1), 103109.

8. Aragón, S., Lapresa, D., Arana, J., Anguera, M. T. \& Garzón, B. (2016). An example of the informative potential of polar coordinate analysis: sprint tactics in elite 1,500-m track events. Meas. Phys. Educ. Exerc. Sci. 21, 2633. doi: 10.1080/1091367X.2016.1245192

9. Blanco-Villaseñor, A., Castellano, J., Hernández-Mendo, A., Sánchez-López, C. R. y Usabiaga, O. (2014). Aplicación de la TG en el deporte para el estudio de la fiabilidad, validez y estimación de la muestra. Revista de Psicología del Deporte, 23(1), 131-137.

10. Cardinet, J. Tourneur, Y., \& Allal, L. (1976). The simmetry of generalizability theory: Applications to educational measurement. Journal of Educational Measurement, 13(2), 119-135. https://doi.org/10.1111/j.17453984.1976.tb00003.x

11. Cardinet, J. Tourneur, Y., \& Allal, L. (1981). Extension of generalizability theory and its applications in educational measurement. Journal of Educational Measurement, 18(4), 183-204. https://doi.org/10.1111/j.17453984.1981.tb00852.x

12. Castañer, M., Barreira, D., Camerino, O., Anguera, M. T., Canton, A. \& Hileno, R. (2016). Goal scoring in soccer: a polar coordinate analysis of motor skills used by Lionel Messi. Front. Psychol. 7:806. doi.org/10.3389/fpsyg.2016.00806

13. Castañer, M., Barreira, D., Camerino, O., Anguera, M. T., Fernandes, T. \& Hileno, R. (2017). Mastery in goal scoring, T-pattern detection, and polar coordinate analysis of motor skills used by Lionel Messi and Cristiano Ronaldo. Front.Psychol. 8:741. doi.org/10.3389/fpsyg.2017.00741

14. Castellano, J. (2000). Observación y análisis de la acción de juego en fútbol. Lecturas: $E F$ $y$ Deportes. Revista Digital, 5(22). Recuperado de http://www.efdeportes.com/efd22b/julentd.ht $\underline{\mathrm{m}}$

15. Castellano Paulis, J. y Hernández Mendo, A. (2002). Aportaciones del análisis de coordenadas polares en la descripción de las transformaciones de los contextos de interacción defensivos en Fútbol. Cronos, 1 , 42-48.

16. Castellano, J. y Hernández-Mendo, A. (2003). El análisis de coordenadas polares para la estimación de relaciones en la interacción motriz en fútbol. Psicothema, 15(4), 569-574.

17. Castellano, J., Perea, A. \& Hernández Mendo, A. (2009). Diachronic analysis of interaction contexts in the 2006 World Cup (212-217), in T. Reilly and Korkusuz, F. (edit.), Science and football VI. London and New York: Routledge.

18. Castellano Paulis, J., Hernández Mendo, A. y Haro Romero, J. A. (2002). Mapas socioconductuales de la selección francesa en el mundial de Francia'98. Revista de Psicología del Deporte,11(1), 35-51.

19. Cochran, W. G. (1954). Some methods for streghning the common $\chi 2$ test. Biometrics, 10, 417-451. doi: $10.2307 / 3001616$

20. Díaz-Díaz, R., Ramos-Verde, E. J., GarcíaManso, J. M., Valverde-Esteve, T.\& ArriazaArdiles, E. (2018). The use of Polar Coordinates in the analysis of motor interaction in football according to the result. Cuadernos de Psicología del Deporte, 19(1), 60-75. 


\section{Quiñones, Morillo-Baro, Reigal, Morales-Sánchez, Vázquez-Diz, Hernández-Mendo.}

21. Echeazarra Escudero, I., Castellano Paulis, J., Usabiaga Arruabarrena, O. y HernándezMendo, A. (2015). Diferencias en el uso estratégico del espacio en categorías infantil y cadete de fútbol: una aplicación del análisis de coordenadas polares. Cuadernos de Psicología del Deporte, 15(1), 169-180. doi.org/10.4321/S1578-84232015000100017

22. Flores, J. y Anguera, M. T. (2018). Patrón de juego en balonmano según el jugador que ocupa la posición de central. Apunts: Educación Física y Deportes, 134(4),110123. doi.org/10.5672/apunts.20140983.cat.(2018/4).134.08

23. García, T., García, J.A., y Aniz, I. (2004). Análisis de la estructura del ataque en equipos de alto nivel de balonmano. Apunts. Educacion Fisica y Deportes, 76 (2), 53-58. https://doi.org/10.5672/apunts.20140983.es.(2015/4).122.01

24. García, J. A., Aníz, I., Arellano, J. I., Domínguez, J. O. y García, T. (2004). Influencia de las variables tiempo y distancia en la eficacia del juego con transformaciones en cuatro equipos de balonmano de alto nivel. Posibilidades para la aplicación en el entrenamiento. Motricidad. European Journal of Human Movement, 12, 79-94 https://doi.org/10.4995/thesis/10251/4624

25. García, J.A., García, T. e Inarejos, J. L. (2002). Análisis de las variables que afectan al juego con desdoblamientos en balonmano y su aplicación al entrenamiento: estudio de un caso en alto rendimiento. Revista de entrenamiento Deportivo, 16,1, 35-40. https://doi.org/10.4321/s1578$\underline{84232012000200008}$

26. González, A. (2012). Análisis de la eficacia del contraataque en balonmano como elemento de rendimiento deportivo (Tesis doctoral). Universidad de León, León, España.
27. González, A., Botejara, J., Puñales, L., Trejo, A. y Ruy, E. (2013). Análisis de la finalización del ataque en partidos igualados en balonmano de alto nivel mediante coordenadas polares. E-Balonmano.com: Revista de Ciencias del Deporte, 9, 71-89.

28. Gorospe, G., y Anguera, M.T. (2000). Modificación de la técnica clásica de coordenadas polares mediante un desarrollo distinto de la retrospectividad: aplicación al tenis. Psicothema, 12(2), 279-282.

29. Hernández-Mendo, A. (1996). Observación y análisis de patrones de juego en deportes sociomotores. (Tesis Doctoral sin publicar). Universidad de Santiago de Compostela, Santiago de Compostela, España.

30. Hernández-Mendo, A., López-López, J., Castellano, J., Morales-Sánchez, V. y Pastrana, J. L. (2012). Hoisan 1.2: Programa informático para uso en metodología observacional. Cuadernos de Psicología del Deporte, $12 \quad$ (1), 55-78. doi.org/10.4321/S1578-84232012000100006

31. López, J. A., Menescardi, C., Estevan, I., Falcó, C. y Hernández-Mendo, A. (2015). Análisis técnico-táctico en Taekwondo con coordenadas polares a través del software HOISAN. Cuadernos de Psicología del Deporte, 15, 131-142. doi.org/10.4321/S1578-84232015000100013

32. Lozano, D. y Camerino, O. (2012). Eficacia de los sistemas ofensivos en balonmano. Apunts: Educación fisica y deportes (108), 70-81. doi.org/10.5672/apunts.20140983.es.(2012/2).108.08

33. Lozano, D., Camerino, O. e Hileno, R. (2016a). Análisis del comportamiento táctico ofensivo en momentos críticos de juego en el alto rendimiento en balonmano: Un estudio Mixed Methods. Cuadernos de Psicología del Deporte, 16(1), 151-160. ISSN 1989-5879 


\section{El juego combinativo ofensivo en el balonmano de élite}

34. Lozano, D., Camerino, O. e Hileno, R. (2016b). Interacción dinámica ofensiva en balonmano de alto rendimiento. Apunts. Educación Física y Deporte, 16(3),90-110. doi.org/10.5672/apunts.20140983.es.(2016/3).125.08

35. Maneiro, R. \& Amatria, M. (2018). Polar coordinate analysis of relationships with teammates, areas of the pitch, and dynamic play in soccer: a study of Xabi Alonso. Front. Psychol. 9:389. doi.org/10.3389/fpsyg.2018.00389

36. Maneiro, R., Amatria, M., Moral, J.E. y López, S. (2018). Análisis observacional de las relaciones interlíneas de la Selección Española de Fútbol, mediante coordenadas polares. Cuadernos de Psicología del Deporte, 18(2), 18-32.

37. Maneiro, R., Amatria, M., \& Anguera, M. T. (2019). Dynamics of Xavi Hernández's game: a vectorial study through polar coordinate analysis. Proc. Inst. Mech. Eng. Part P J. Sports Eng. Technol. doi.org/10.1177/1754337119830472

38. Meletakos, P., Vagenas, G., \& Bayios, I. (2011). A multivariate assessment of offensive performance indicators in Men's Handball: Trends and differences in the World Championships. International Journal of Performance Analysis in Sport, 11(2), 284294.

doi.org/10.1080/24748668.2011.11868548

39. Montoya, M., Moras, G. y Anguera, M. T. (2013). Análisis de las finalizaciones de los jugadores extremo en balonmano. Apunts: Educación Física y Deportes, 113, 52-59. doi.org/10.5672/apunts.20140983.es.(2013/3).113.05

40. Morgado, A. P. (2012). Análisis de los factores de eficacia de las acciones de prefinalización y finalización en ataque organizado en balonmano de alto nivel. (Tesis doctoral). Universidad de Castilla-La Mancha. Toledo, España. https://doi.org/10.4995/thesis/10251/54108

41. Morillo Baro, J.P. y Hernández-Mendo, A. (2015). Análisis de la calidad del dato de un instrumento para la observación del ataque en balonmano playa. Revista Iberoamericana de Psicología del Ejercicio y el Deporte. 10(1), 15-22.

42. Morillo, J. P., Reigal, R. E. y HernándezMendo, A. (2015). Análisis del ataque posicional de balonmano playa masculino y femenino mediante coordenadas polares. Rev. Int.Cien. Deporte 11, 226-244. doi.org/10.5232/ricyde2015.04103

43. Morillo, J.P., Reigal, R.E., HernándezMendo, A., Montaña, A. \& Morales-Sánchez, V. (2017). Decision-Making by Handball Referees: Design of an ad hoc Observation Instrument and Polar Coordinate Analysis. Front. Psychol. doi.org/10.3389/fpsyg.2017.01842

44. Navarro, A., Morillo-Baro, J. P., Reigal, R. E. \& Hernández-Mendo, A. (2018). Polar coordinate analysis in the study of positional attacks in beach handball. Int. J. Perf. Anal. Sport 18, 151-167. doi.org/10.1080/24748668.2018.1460052

45. Nunes, H., Iglesias, X., Daza, G., Irurtia, A., Caparros, T. y Anguera, M.T. (2015). Influencia del pick and roll en el juego de ataque en baloncesto de alto nivel. Cuadernos de Psicología del Deporte,16(1), 129-142.

46. Prudente, J., Sousa, D., Sequeira, P., LópezLópez, J. A. \& Hernández-Mendo, A. (2017). Analyzing the influence of playing time and partial score on the tactical behavior in the duel 2 vs 2 in the offensive process in handball, using the polar coordinates technique. Anales de psicología, 33(3), 515529. doi.org/10.6018/analesps.33.3.271071 


\section{Quiñones, Morillo-Baro, Reigal, Morales-Sánchez, Vázquez-Diz, Hernández-Mendo.}

47. Quiñones, Y., Morillo-Baro, J. P., Reigal, R. E., Morales-Sánchez, V., Vázquez-Diz, J. A. y Hernández-Mendo, A. (2019). El ataque posicional en balonmano: validación de un sistema de observación. Cuadernos de Psicología del Deporte, 19(3), 114-124. doi.org/10.6018/cpd.384091

48. Riveiro-Bozada, A., García-García, O., Serrano-Gómez, V., Morales-Sánchez, V., López-López, J. A. y Hernández-Mendo, A. (2016). Influencia del nivel de competición en las acciones técnicas de punto realizadas en Shiai Kumite femenino de karate. Análisis de coordenadas polares. Cuadernos de Psicología del Deporte, 16(1), 51-68.

49. Sackett, G. P. (1980). "Lag sequential analysis as a data reduction technique in social interaction research," in Exceptional Infant. Phychosocial Risks in InfantEnvironment Transactions, eds D. B. Sawin, R. C. Hawkins, L. O. Walker and J. H. Penticuff (New York, NY: Brunner/Mazel), 300-340.

50. Sousa, D. J., Prudente, J., Sequeira, P., LópezLópez, J. A. y Hernández-Mendo, A. (2014). Análisis de las situaciones de juego 2vs2 en el campeonato europeo masculino de balonmano 2012: Aplicación de la técnica de coordenadas polares. Cuadernos de Psicología del Deporte, 5, 181-194. doi.org/10.4321/S1578$\underline{84232015000100018 .}$.

51. Tarragó, R., Iglesias, X., Lapresa, D. \& Anguera, M. T. (2016). Complementariedad entre las relaciones diacrónicas de los $\mathrm{T}$ Patterns y los patrones de conducta en acciones de esgrima de espada masculina de élite. Cuadernos de Psicología del Deporte. 16, 113-128.

52. Tarragó, R., Iglesias, X., Lapresa, D., Anguera, M.T., Ruiz-Sanchís, L. \& Arana, J. (2017). Analysis of diachronic relationships in successful and unsuccessful behaviors by world fencing champions using three complementary techniques. Anales de Psicología, 33(3), 471-485. doi.org/10.6018/analesps.33.3.271041

53. Teles, N. J. (2011). Influência das variáveis contextuais na performance das equipas nos últimos dez minutos do jogo de Andebol. (Tesis doctoral), Universidad Técnica de Lisboa, Lisboa, Portugal.

54. Vázquez-Diz, J. A., Morillo-Baro, J. P., Reigal, R. E., Morales-Sánchez, V. y Hernández-Mendo, A. (2019a). Diseño y validación de una herramienta de observación para porteros en balonmano playa. Cuadernos de Psicología del Deporte, 19(2), 135-146.

55. Vázquez-Diz, J. A., Morillo-Baro, J. P., Reigal, R. E., Morales-Sánchez, V. \& Hernández-Mendo, A. (2019b). Mixed Methods in Decision-Making Through Polar Coordinate Technique: Differences by Gender on Beach Handball Specialist. Front. Psychol. 10:1627. doi.org/10.3389/fpsyg.2019.01627

56. Vázquez-Diz, J. A., Morillo-Baro, J. P., Reigal, R. E., Morales-Sánchez, V. \& Hernández-Mendo, A. (2019c). Contextual Factors and Decision-Making in the Behavior of Finalization in the Positional Attack in Beach Handball: Differences by Gender Through Polar Coordinates Analysis. Front. Psychol. 10:1386. doi.org/10.3389/fpsyg.2019.01386 